\title{
Multiple asteroid systems from the UkrVO digitized photographic plates
}

\author{
Kazantseva $\mathrm{L}^{* 1}$ and Shatokhina $\mathrm{S}^{\dagger 2}$ \\ $1_{\text {Taras Shevchenko National University of Kyiv, Ukraine, Kyiv, Ukraine }}$ \\ ${ }^{2}$ Main Astronomical Observatory of the NAS of Ukraine, Kyiv, Ukraine
}

\begin{abstract}
Several catalogs of various celestial objects were compiled based on the processing of Ukrainian VO archives and observatories in Kitab (Uzbekistan) and Baldone (Latvia). We analyzed the catalogs of asteroid positions and B-magnitudes obtained from the processing of digitized photographic plates and selected from them asteroids, which are known as multiple. Positional observations cover the period 1973-1993 and may be useful for a more detailed study of the dynamics of these systems. The obtained photometric characteristics of asteroids will be useful for studying changes in brightness.
\end{abstract}

Keywords: Double asteroids, positional catalog, digitized photographic observations

\section{Introduction}

The first observations indicating the possible presence of satellites in asteroids were obtained in 1977 (visual observation of stellar occultation of minor planet 6 Hebe) and 1978 (photometric light curve observation of asteroid 532 Herculina). But in the absence of other observational evidence, the existence of multiple asteroids and asteroids with satellites remained likely until space missions. Since then, the search for asteroid multiplicity by various methods (Hubble Space Telescope, observations with ground-based telescopes, radar, etc.) has begun.

As of October 2020, 416 such celestial bodies are already known (Johnston (2018)). 42 of them were detected from ground-based observations, 97 from space-based surveys, 49 by radar, 227 from the analysis of photometric curves. These are 400 double systems, 15 triple. Also recently, this group of objects includes a sextuple system of Pluto. In total, these small bodies have 435 satellites. According to the types of orbits, they are divided into near-Earth (78), main-belt asteroids (187), those that cross the orbit of Mars (31), the Trojan asteroids of Jupiter (6), and trans-Neptune (114). Also, among them, there is 1 object with rings and 1 object with signs of a double comet.

But the main feature of this extremely interesting new group of celestial bodies is that they are still insufficiently studied. According to the type and level of confirmation, only 48 of them are currently available for observation, 27 already have certain parameters, 126 are considered confirmed, and 215 are still probable.

Therefore, any new observational data on these objects is very important extensive work on the processing of photographic plates, obtained in 1969-1995, was carried out by MAO NASU (Vavilova et al. (2012)) in cooperation with the observatories participating in the Northern Sky Survey (FON) project.

\footnotetext{
*kazantsevaliliya1@gmail.com, Corresponding author
}

${ }_{\dagger}^{\dagger}$ svetash@mao.kiev.ua 

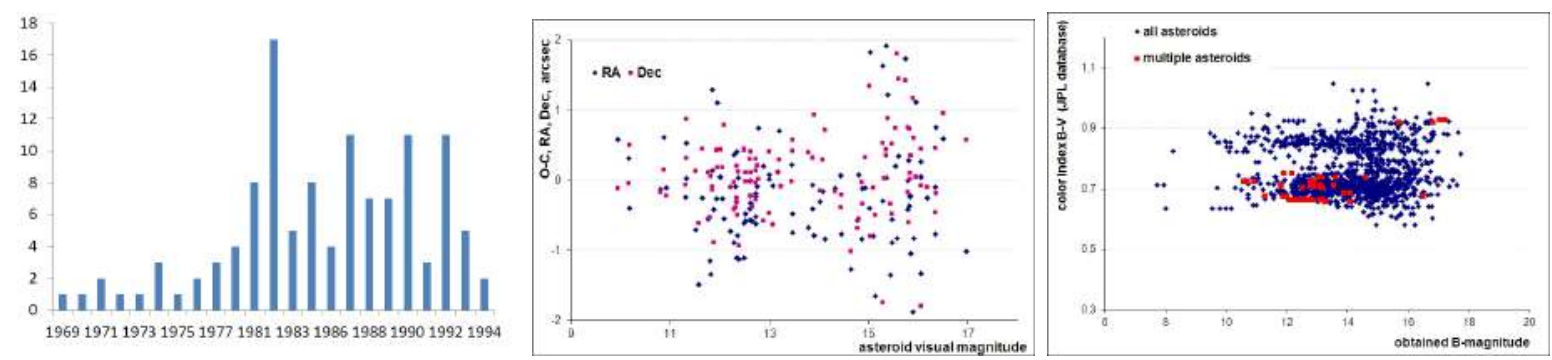

Figure 1. Distributions of selected multiple asteroids by observation time (left), by coordinate differences $\mathrm{O}-\mathrm{C}$ (in the middle) and B-magnitudes in comparison with all identified asteroids from FON-Kyiv part of the project

\section{Main results}

Several catalogs of coordinates and B-magnitudes for more than 32 million stars and galaxies have been created based on the processing of Kyiv and Kitab digitized observations of the FON project ( Andruk et al. (2016), Andruk et al. (2017), Yuldoshev et al. (2018), Yuldoshev et al. (2019)). Becides, a few catalogs of coordinates and B-magnitudes of asteroids and comets were compiled based on these data (Shatokhina et al. (2018b), Shatokhina et al. (2018a), Shatokhina et al. (2019)). A preliminary catalog of asteroid positions and magnitudes was compiled based on digitized observations of the open star clusters in UBVR color bands obtained at the Baldone observatory (Latvia) in 1967-1995 (Eglitis et al. (2019)).

The coordinates determined in the Tycho-2 reference system, and the B-magnitudes are obtained in the system of photoelectric standards. Digitization of photographic plates was carried out using Epson $^{T M}$ and Microtek ${ }^{T M}$ commercial scanners, with scanning mode - 1200 dpi, linear image size $30 \mathrm{x} 30 \mathrm{~cm}$ or $13000 \mathrm{x} 13000 \mathrm{px}$.

We have analyzed 5020 positions of asteroids on digitized observations of the Kyiv and Kitab parts of the FON project and 1872 previous positions in UBVR spectral bands on digitized observations in Baldone. As a result, 119 positions were selected for 69 asteroids, which are multiple asteroid systems or asteroids with companions according to the databases (http://cbat.eps.harvard.edu/minorsats, http://www.johnstonsarchive.net/astro/asteroidmoons). Most of them belong to the main belt asteroids, three of them belong to the Jupiter Trojans family, 4 are Mars crossers and 1 are near- Earth object. According to these data, positions were obtained from observations of the period 1969-1994. The all positions were compared with the JPL ephemeris and the O-C differences calculated, shown in Fig. 1. The figure also shows the obtained B-magnitudes of multiple asteroids depending on their color indices in comparison with all identified asteroids from the FON-Kiev part of the project. Both bright asteroids and faint ones were selected. Given that the average positional accuracy of determining coordinates and B-values for bright $11-13^{m}$ is twice better than for faint $11-16^{m}$ asteroids (Shatokhina et al. (2018a)), this may be essential for determining some kinematic and photometric characteristics these asteroids.

\section{Conclusion}

From catalogs of asteroid positions and magnitudes based on the processing of digitized photographic observations of the FON project and the Baldone archive we selected and analyzed 119 positions and magnitudes of multiple asteroid systems. Considering that this group of multiple asteroids has not been sufficiently studied, any of their positional observations can be useful for studying the dynamics of their systems. And the magnitudes obtained simultaneously with their coordinates can be used to construct and analyze the light curves and other photometric characteristics of these asteroids. 


\section{References}

Andruk V. M., Pakuliak L. K., Golovnia V. V., et al. 2016, Kinem. Phys. Cel. Bodies, 32, 260

Andruk V. M., Pakuliak L. K., Golovnia V. V., et al. 2017, VizieR Online Data Catalog, p. I/342

Eglitis I., Yizhakevych O., Shatokhina S., et al. 2019, Odessa Astron. Publ., 32, 189

Johnston W., 2018, NASA Planetary Data System,

Shatokhina S. V., Relke H., Yuldoshev Q., et al. 2018a, Odessa Astron. Publ., 31, 235

Shatokhina S. V., Kazantseva L. V., Yizhakevych O. M., et al. 2018b, Kinem. Phys. Cel. Bodies, 34, 270

Shatokhina S. V., Kazantseva L. V., Yizhakevych O. M., et al. 2019, VizieR Online Data Catalog (other), 0030, J/other/KFNT/34

Vavilova I. B., Pakulyak L. K., Shlyapnikov A. A., et al. 2012, Kinem. Phys. Cel. Bodies, 28, 85

Yuldoshev Q. X., Ehgamberdiev S. A., Muminov M. M., et al. 2018, VizieR Online Data Catalog, p. $\mathrm{I} / 346$

Yuldoshev Q., Protsyuk Y., Relke H., et al. 2019, Astron. Nachr., 340, 494 